\title{
Topological Features of Hot Carrier Induced Anisotropic Breakdown on Silicon Diode Surfaces
}

\author{
George G. Harman \\ Institute for Applied Technology, National Bureau of Standards, Washington, D.C. 20234
}

(February 11, 1969)

\begin{abstract}
Both Gunn and Morozov have reported breakdown paths (tracking) on the surface of germanium under hot carrier conditions. Many silicon and gallium arsenide device failures appear to have been caused by similar breakdown tracks extending between contacts or across junctions. In the present work on silicon, extremely anisotropic tracking has been observed on the surface of long, thin, forward biased, $n^{+}-p-p^{+}$, silicon diodes. The tracks propagate only in $\langle 100\rangle$ crystallographic directions, independent of the applied field orientation, the temperature, or the crystal growth direction. For example, on a sample with a $\{100\}$ surface plane, having the field oriented along a $\langle 110\rangle$ direction, the tracks propagate along $\langle 100\rangle$ directions which are $45^{\circ}$ away from the applied field. Tracks on $\{100\}$-plane, disk shaped diodes, propagate radially from the center (positive biased) ohmic contact region, and mark off the $\langle 100\rangle$ directions within $2^{\circ}$. Tracking requires both high current densities $\left(\sim 5 \times 10^{3} \mathrm{~A} / \mathrm{cm}^{2}\right)$ and high fields $(\sim 15 \mathrm{kV} / \mathrm{cm})$, and occurs most readily on the $p$-region of $10 \Omega \mathrm{cm}$, $n^{+}-p-p^{+},\langle 100\rangle$ oriented diodes. Diodes with $n^{+}-n-p^{+}$structures having $n$-regions $\sim 1 \Omega \mathrm{cm}$, and $n^{+}-n-n^{+}$structures of $\sim 1 \Omega \mathrm{cm}$ will also track, but require much higher fields and the $\langle 100\rangle$ tracking orientation is not clearly defined. Tracking does not occur on diodes having the field oriented along a $\langle 111\rangle$ direction.

Two basic types of tracks are observed. The first resembles a series of tiny explosion craters $(\sim 10 \mu \mathrm{m}$ diam). The second appears to be continuous in nature, even though it is extended by each applied pulse. These tracks may be several micrometers wide and deep.

It is also shown that hot-minority-carrier sample explosions are anisotropic and not, as generally assumed, caused by thermal breakdown. Typically a $\{110\}\langle 111\rangle$ oriented $n^{+}-p-p^{+}$sample requires 4 to 6 times more impulse energy to explode than a $\{100\}\langle 100\rangle$ oriented sample.
\end{abstract}

Key words: Anisotropy; crystallographic orientation; hot carriers; semiconductor breakdown; silicon diodes.

\section{Introduction}

In early hot carrier experiments Gunn [1] ${ }^{1}$ observed "visible tracks" on the surface of his $n$-type germanium structures, when the fields were $\sim 10 \mathrm{kV} / \mathrm{cm}$. No crystallographic orientation of his samples was reported and presumably the tracks were in the general direction of the applied field, originating at and extending from the electrodes. He found it necessary to shorten the pulse length to $\sim 10^{-9} \mathrm{~s}$ in order to apply high fields without damage to his samples.

Morozov [2] observed oriented breakdown on germanium surfaces, extending from point contacts. He applied long $(10-1000 \mu \mathrm{s})$, high current (up to $30 \mathrm{~A}$ peak) pulses by discharging a capacitor through the point contacts. Tracks were observed only when the point was positive, regardless of the carrier type. There was some evidence of molten material in the tracks. No absolute orientation preference was observed. However, at high power levels the breakdown generally followed the crystal growth direction, which was a $\langle 111\rangle$.

Considering this history plus the fact that, com-

\footnotetext{
${ }^{1}$ Figures in brackets indicate the literature references at the end of this paper.
}

mercial semiconductor devices often operate with fields as high or higher than the above hot carrier structures, it was decided to investigate the occurrence of the tracking phenomena on silicon, characterize it, and if possible relate it to various device breakdowns. Some preliminary results of this work have been re. cently reported $[3,4]$.

In the following work we will describe some of the high field, high current transport properties, including conductivity modulation, of long thin forward biased $n^{+}-p-p^{+}$silicon diode structures. The hot carrier excitation produces surface breakdown tracks that propagate along $\langle 100\rangle$ crystallographic directions, regardless of the field orientation. The general morphology of the two basic types of tracks will be described in detail. The average applied (and local) field and current density thresholds necessary to produce tracking will be given, and the effects of differing bulk carrier type and concentrations discussed. At very high impulse power levels all of the samples will explode. However, the power level for explosions is strongly dependent on the crystallographic orientation of the applied field. Various mechanisms that might result in such effects are discussed. 
lographic orientation of the applied field. Various mechanisms that might result in such effects are discussed.

\section{Apparatus and Sample Preparation}

High voltage pulses were generated with a hydrogen thyratron delay-line system operating into $25 \Omega$ impedance, similar to that described in reference 4 . Pulse lengths could be varied from 0.06 to $3.0 \mu \mathrm{s}$. A $0.4 \mu \mathrm{s}$ pulse length was used for most of the measurements reported here. The system could deliver up to $8 \mathrm{kV}$ directly or could be stepped up to $25 \mathrm{kV}$ through a specially designed pulse transformer. High voltage diode stacks were used as reverse polarity clippers both in the generator and on the transformer output, in order to protect the reverse characteristics of the junctions. The system was generally terminated within $2 \mathrm{~cm}$ of the sample using several $2 \mathrm{~W}$ carbon resistors in series, immersed in the same insulating liquid as the sample, either liquid nitrogen or a fluorocarbon. The current was measured either with a precision current transformer or across a $2 \Omega$ load in series with the sample.

The long thin $p-n$ junction samples present a nonlinear "inductive" load with an equivalent inductance of 30 to $50 \mu \mathrm{H}$. The current through the sample rises as a sawtooth from zero to, at times, greater than 40 A peak. This caused matching problems not encountered in the usual hot majority-carrier studies. Even with $25 \Omega$ source impedance it was difficult to maintain a good square voltage waveshape at all times, so the reported pulse voltages and currents were measured at the same point in time, at the current peak on the end of the pulse. A very low repetition rate (typically 4 pps or less) was used, to assure that the sample temperature returned to the ambient value between pulses.

In order to determine the field distribution along the samples, a pulse probe apparatus was developed. This consisted of a tuned, high voltage oscilloscope probe with an internal impedance of $10^{7} \Omega$. A coarse phosphor-bronze point contact $(\sim 7 \mu \mathrm{m}$ diam tip) was attached to the end of the probe. This was then mounted on a micromanipulator platform and lowered onto the sample, using a microscope for alinement. The probe gave accurate voltage pulse shapes regardless of whether it was forward or reverse biased (through its $10^{7} \Omega$ internal impedance). In the reverse direction such a coarse point results in low breakdown voltages and high leakage currents, so that no effective barrier is observed. Since the sample impedances were typically less than $10^{3} \Omega$, no probe induced perturbation of the internal field was anticipated. A comparative test with a $10^{8} \Omega$ probe yielded identical results.

The samples discussed in this paper were $1,7,10$, and $100 \Omega \mathrm{cm}$ silicon of both $n$ and $p$ type, cut in different orientations from a small number of low oxygen content, float zone crystals having a $\langle 111\rangle$ growth direction. For verification, samples obtained from different sources and having different growth techniques and directions were also used. Most of the samples were taken from silices cut with various orientations in $\{100\}$ and $\{110\}$ planes, but a few were prepared from $\{111\}$ plane slices [5]. Figure 1 shows the two sample configurations used in this work with the typical dimensions and orientations. The dimensions were varied somewhat for specific experiments. The rectangular (linear) samples (fig. 1a) were variously prepared by an ultrasonic cutting process, or by milling (with a wide diamond wheel) a $0.25 \mathrm{~cm}$ wide, $0.01 \mathrm{~cm}$ deep indentation in the silicon slice at the desired orientation angle, then diamond sawing each sample out. Twenty to thirty samples, having various orientations could be obtained from one slice. This assured a minimum variation of material parameters from sample to sample so that the various comparisons would be meaningful. The disk samples (fig. lb) were made by a two-step ultrasonic cutting process.

During processing all masking and etching was done individually by hand but the diffusions were made in as large numbers as practical to minimize sample to sample variations. The samples were etched, at least $50 \mu \mathrm{m}$ on all sawed or abraded surfaces, to minimize work-damage-induced surface defects. Thus, the final surfaces were not mirror finishes but had the slight "orange peel" configuration that is typical of heavily etched silicon.

Ôhmic contacts on the $p$-type samples were either alloyed aluminum or diffused boron, over-evaporated with aluminum. No significant electrical differences
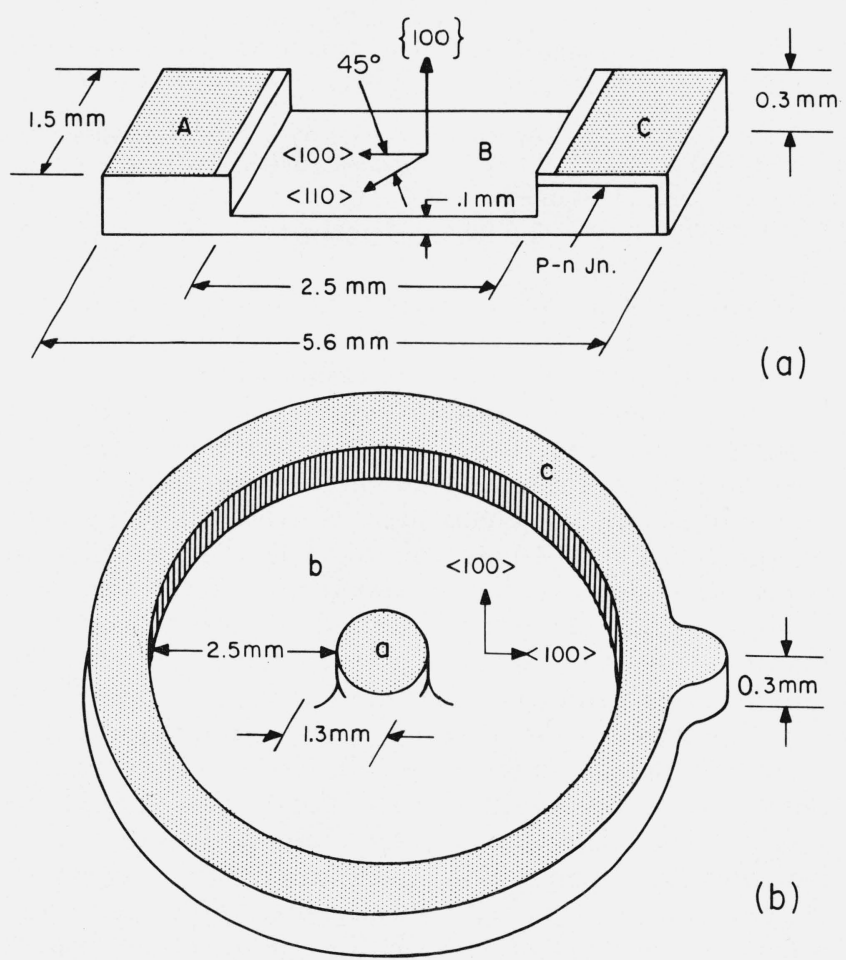

FIGURE 1. Typical sample configurations with the most usual orientations indicated.

Regions $B$ and $b$ are the same thickness. The surface plane orientations are $\pm 1 / 2$ degree, directions are within 2 deg. A, a, are ohmic electrodes; B, b, high field region; C, c, p-n junction region. 


\section{Experimental Observations}

\subsection{Transport}

The current and voltage characteristics of typical forward biased $\langle 100\rangle$ oriented linear diodes are given in figure 2 for both 77 and $300 \mathrm{~K}$ sample temperatures (curves $\mathrm{A}$ and $\mathrm{B}$ ). Current and voltage tracking thresholds are indicated at the top and a typical current wave shape $(\mathrm{E})$, due to injection, is at the bottom. The lower curves (C and D) are for samples with noninjecting, ohmic contacts, at $300 \mathrm{~K}$. These curves, and comparable ones taken at $77 \mathrm{~K}$, show the typical current saturation expected of hot majority carriers. Comparison of curves $\mathrm{C}$ and $\mathrm{D}$ with $\mathrm{B}$, demonstrates the very large conductivity modulation due to minority carrier injection. It is greater than a factor of ten. With the accompanying carrier velocity saturation, it is clear that this increased current density represents increased carrier density. The slopes (S) of various curves are indicated. The slopes of curves $A$ and $B$ increase up to about 2 with increasing pulse width. However, thermal effects become significant for pulse lengths $\sim 1 \mu \mathrm{s}$ and the slope actually decreases in the high peak power region. Very short pulse lengths $(<0.1 \mu \mathrm{s})$ do not result in appreciable injection, so that such curves, and tracking characteristics, resemble those of ohmic contacted samples, to be described below. In curve B, the current wave

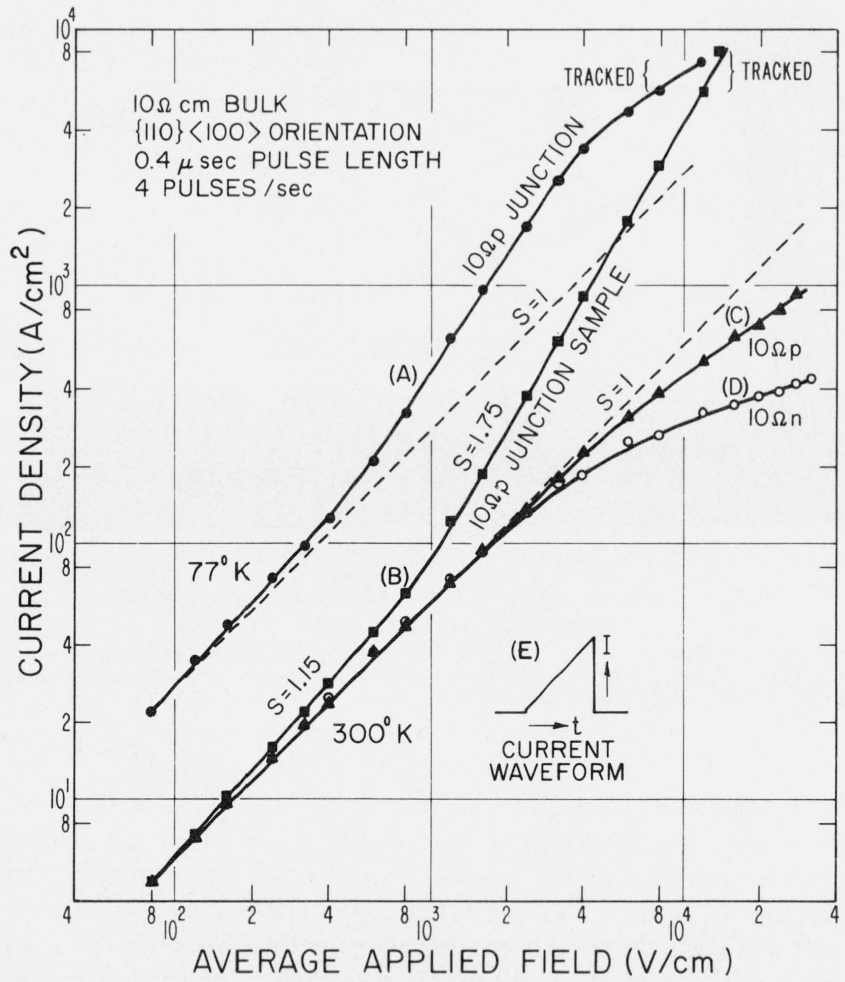

FIGURE 2. The J-E characteristics of typical $10 \Omega \mathrm{cm} \mathrm{n}^{+}-\mathrm{p}-\mathrm{p}^{+}$, forward biased, $\langle 100\rangle$ oriented linear diodes are given for 77 (curve $A$ ) and $300 \mathrm{~K}$ (curve B) sample temperatures.

Tracking current and voltage thresholds are indicated at the top. The lower curves are ting, ohmic contacts; (C) $10 \Omega \mathrm{cm} p^{+}-p-p^{+}$, at $300 \mathrm{~K}$, (D) $10 \Omega \mathrm{cm}$ , and $(\mathrm{E})$ is the current wave shape in the $S=1.75$ region of curve $\mathrm{B}$, resulting from heavy injection. shape of the 1.15 slope portion approximately follows the applied voltage squarewave pulse, indicating that few, if any, injected carriers are able to traverse the entire length of the sample before they recombine. The change of slope to 1.75 is accompanied by the sawtooth current wave shape $(\mathrm{E})$ and is due to minority carrier transport through to the ohmic contact. This latter fact has been verified by infrared $(1.1 \mu)$ photographs of recombination radiation at the ohmic contact, and by studying the voltage wave shape along the sample, using a probe.

The high current-density short pulse-conduction is not in equilibrium. However, it appears to be a case of double injection when the data is modified for the saturating velocities of the carriers, taking into account the effects of diffusion at the ends, by the method of Mayer, Baron and Marsh [6].

\subsection{Anisotropic Tracking}

When the voltage and current are increased through a forward biased long thin $n^{+}-p-p^{+}$diode, the first evidence of anything unusual is that short, weak pulses of avalanche radiation are emitted [4]. This is a form of prebreakdown light and can be detected with a photomultiplier. The intensity of these light pulses increases with current until at some point $(\sim 3$ to 10 times threshold current, depending on orientation) there will appear very intense, fractional microsecond bursts of light that are visible with the dark accommodated eye and can saturate a photomultiplier. These occur simultaneously with the peak of the current pulse (fig. $2 \mathrm{E}$ ) and are the first evidence that tracks have started. At this time there is no indication on the current envelope itself. After an extended period of pulsing, the current envelope may become erratic at the peak (the top $5 \%$ of the total current). By the time this is seen, several tracks would have started or a tiny chip may have been blown from the sample.

On $n^{+}-p-p^{+}$silicon structures, the tracks are always oriented in a $\langle 100\rangle$ crystallographic direction. Thus for samples having applied fields along the $\langle 100\rangle$ direction the tracks will be parallel to the field. They originate in the high field region (B of fig. la), near the positively biased ohmic (noninjecting) contact end of the sample and propagate toward the negatively biased junction, progressing at a rate of from 1 to $10 \mu \mathrm{m}$ per pulse, depending on the applied power level. The tracks will reach a maximum length for any given power level, and continued pulsing will only widen and deepen them. Relatively large surface imperfections produce only microscopic deviations in the direction of propagation of the track.

Figure 3 shows a sample with the field oriented in a $\langle 110\rangle$ direction, having a $\{100\}$ surface plane. The breakdown tracks are clearly at $45^{\circ}$ from the direction of the applied field and thus are in a $\langle 100\rangle$ direction. Figure 4 shows a section of a silicon web sample

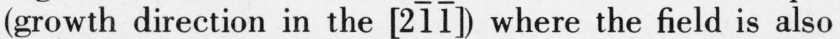
in a $\langle 110\rangle$ direction, but the surface plane is a $\{111\}$. No $\langle 100\rangle$ direction exists on this surface but the projection of a $\langle 100\rangle$ lies at an angle of $30^{\circ}$ from the $\langle 110\rangle$ 


\section{$\langle 110\rangle \longrightarrow$}

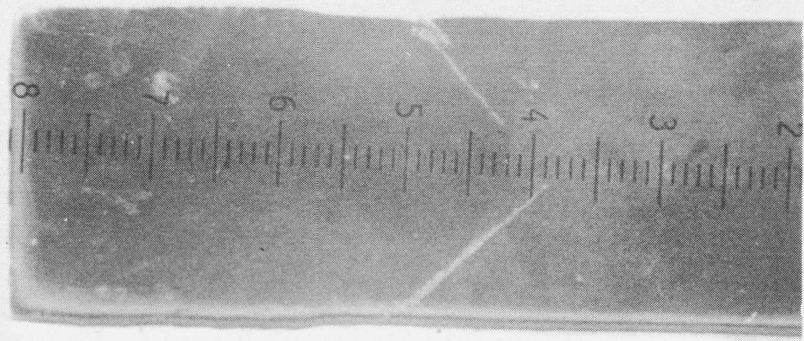

FigURE 3a. A $10 \Omega \mathrm{cm}$, p-type $\{100\}\langle 110\rangle$ oriented, linear junction sample, showing breakdown tracks at $45^{\circ}$ from the applied field direction and thus in the $\langle 100\rangle$ direction.

Breakdowns initiated and propagated on the back side (away from electrodes). The tracking threshold was $26 \mathrm{kV} / \mathrm{cm}, 22 \times 10^{3} \mathrm{~A} / \mathrm{cm}^{2}, 0.5 \mu$ s pulse length, at $300 \mathrm{~K}$.

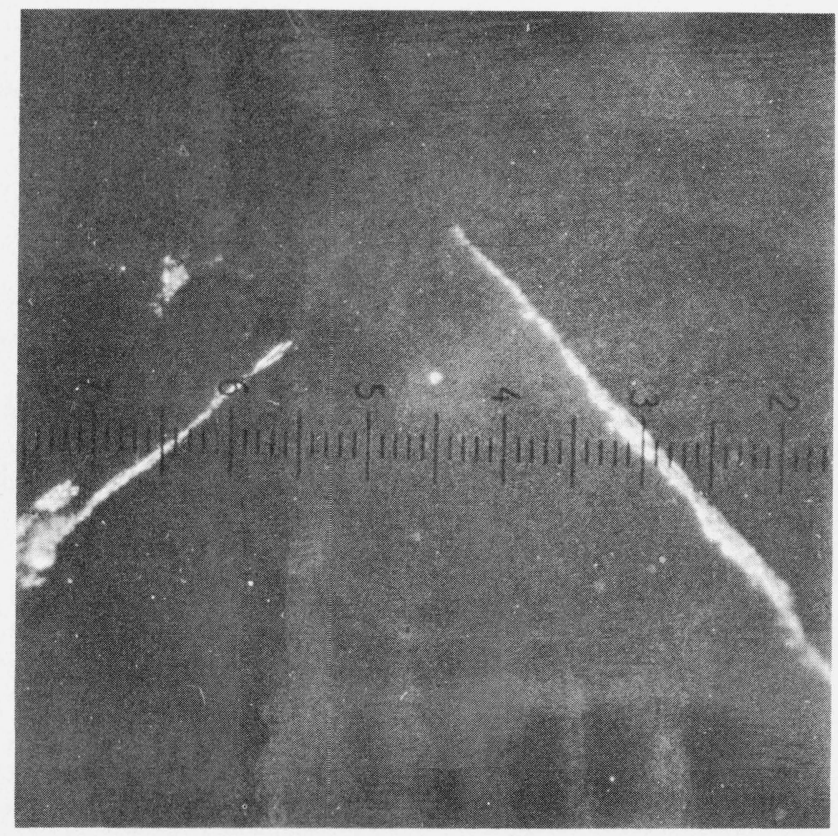

FIGURE 3b. A larger magnification of the same sample emphasizing the $45^{\circ}$ tracks.

and this is the angle the track made with respect to the applied field. Thus in the preceding two figures we have seen two different tracking directions with the field in the same direction, but with crystals having different surface planes. Returning to the $\{100\}$ surface plane, but this time with the field applied $22^{\circ}$ away from a $\langle 100\rangle$ direction, we find in figure 5 that the track appears at an angle of $22^{\circ}$ from the applied field direction.

The $\langle 100\rangle$ track direction has now been established for linear samples. However, the ultimate proof of

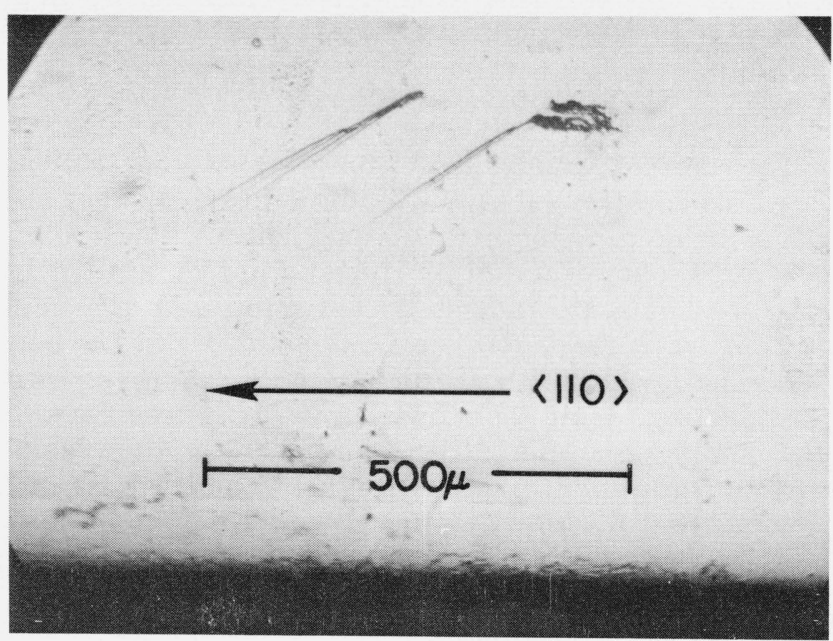

FigURE 4. A portion of a $7 \Omega \mathrm{cm}$ p-type $\{111\}\langle 110\rangle$ oriented linear junction sample (made from silicon web) showing the breakdown tracks at $30^{\circ}$ from the applied field direction.

The tracks started in the high field region (B of fig. la), far away from the electrodes. The tracking threshold was $25 \mathrm{kV} / \mathrm{cm}-8.5 \times 10^{3} \mathrm{~A} / \mathrm{cm}^{2}, 0.25 \mu$ s pulse length, at $77 \mathrm{~K}$.

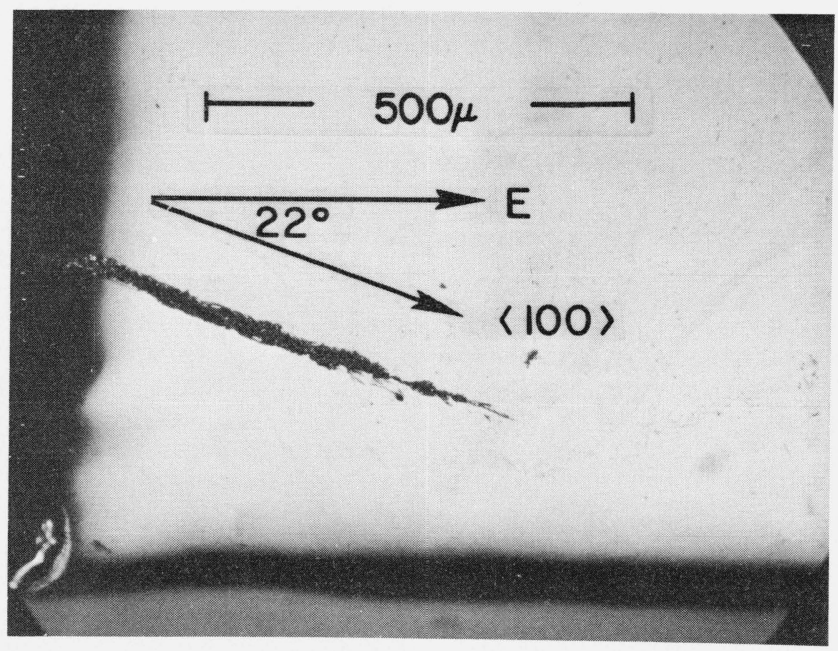

FIGURE 5. A portion of a $10 \Omega \mathrm{cm},\{100\}$ junction sample with the field oriented $22^{\circ}$ from the $\langle 100\rangle$ direction.

The track originates on the thin, high field region in the shadow of the raised ohmic electrode and is at $22^{\circ}$ from the applied field direction. The tracking threshold was $20 \mathrm{kV} / \mathrm{cm}$, $4 \times 10^{3} \mathrm{~A} / \mathrm{cm}^{2}, 0.4 \mu$ s pulse length, at $300 \mathrm{~K}$.

the orientational preference is given by a disk type sample (fig. 1b) where the field is equal in all directions on the $\langle 100\rangle$ surface plane of the sample. In figure 6 it can be seen that the tracks clearly mark off a $\langle 100\rangle$ axis. An even more definitive case is presented by the disk diode of figure 7 . Here the alloyed aluminum making the center (ohmic) electrode was allowed to extend down the edge of the central mesa and slightly onto the flat region, in a $\langle 110\rangle$ direction. This distorted the field and the track initiated in a $\langle 110\rangle$ direction, but it quickly changed to a $\langle 100\rangle$. 


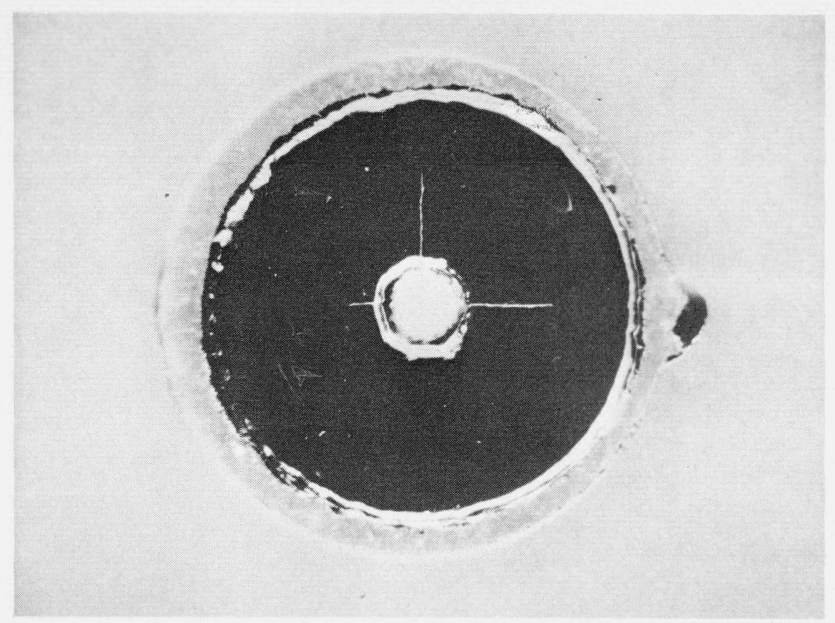

Figure 6. A $10 \Omega \mathrm{cm}$, p-type, $\{100\}$ plane, disk sample with the $\mathrm{p}-\mathrm{n}$ junction on the outer rim and the ohmic contact in the center.

The tracks originate in the high field region at the base of the raised ohmic contact, and propagate in $\langle 100\rangle$ directions only. The applied power to produce tracking was $1500 \mathrm{~V}$, $6.5 \mathrm{~A}, 0.25 \mu \mathrm{s}$ pulse length, at $77 \mathrm{~K}$.

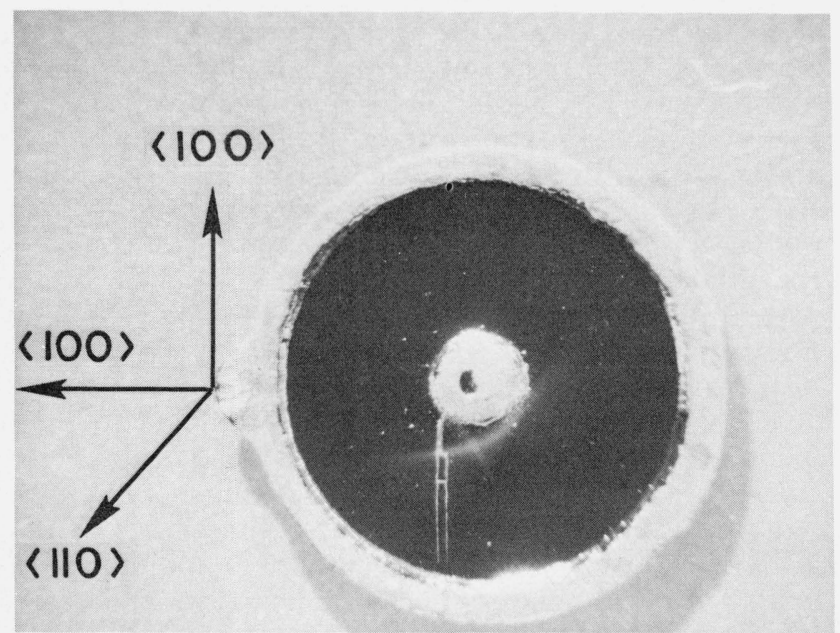

FigURE 7. A $10 \Omega \mathrm{cm}, \mathrm{p}$-type, $\{100\}$ plane, disk sample with the junction on the rim and the ohmic contact in the center.

The alloyed aluminum contacting the center electrode extended over the edge in a $\langle 110\rangle$ direction. The indicated orientations are all in the plane of the sample.

Another interesting point is the development of the second major track with a cross coupling. Except for the initial breakdown, all tracks are nonradial but are nevertheless in $\langle 100\rangle$ directions, including the cross linkage, which appears to have propagated against the field!

In contrast to the $\langle 100\rangle$ orientation observed on p-type bulk when minority carriers are present, tracking occurs in strongly branched, random directions in their absence, as seen in figure 8 . Such tracks are primarily influenced by the orientation of the applied field rather than the crystal structure. In addition the tracking threshold energy is much higher, very near thermal breakdown. Figure 8 is a picture of a $\langle 100\rangle$ oriented $1 \Omega \mathrm{cm}$-type ohmic-contacted sample.

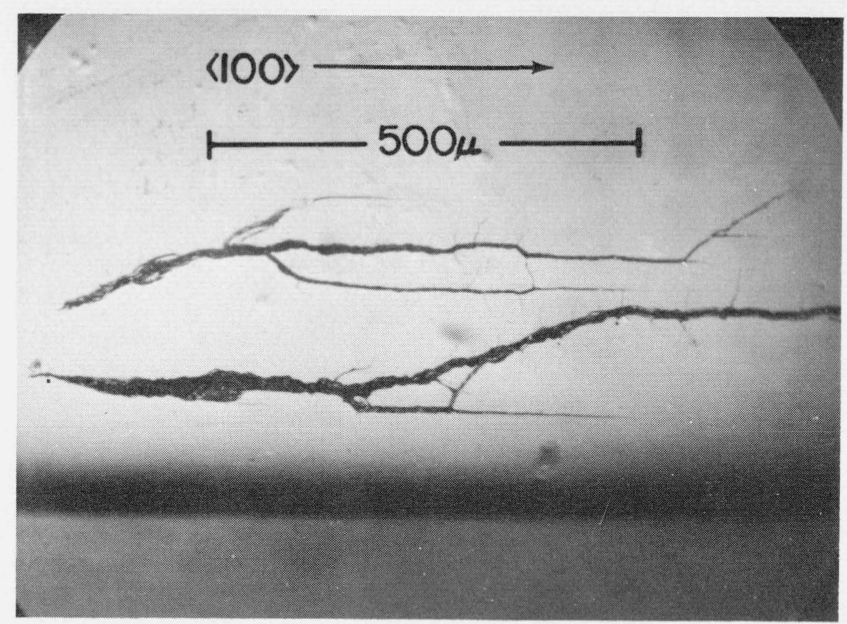

FIGURE 8. Unoriented tracking on a $1 \Omega \mathrm{cm}$ p-type $\{100\}\langle 100\rangle$ linear sample, having diffused boron, ohmic contacts.

The tracks started in the high field region B, far away from the electrodes. The tracking threshold was $30 \mathrm{kV} / \mathrm{cm}, 12.5 \times 10^{3} \mathrm{~A} / \mathrm{cm}^{2}, 0.4 \mu$ s pulse length, at $300 \mathrm{~K}$.

Similar unoriented results are obtained from $1 \Omega \mathrm{cm}$ $n$-type ohmic specimens. No tracking (either oriented or as in fig. 8) is observed on $10 \Omega \mathrm{cm} n$ - or $p$-type ohmic contacted samples, presumably because the necessary high current density cannot be obtained (see fig. 2 curves C and D).

\subsection{Morphology of the Tracks}

There are two basic types of tracks. The first we shall call the explosion type and the second continuous. The first and most common type appears to propagate by a series of tiny explosions each one of which blows out a small crater $\sim 10 \mu \mathrm{m}$ deep and of similar diameter. Such a track is clearly shown in figure 9. The track initiated near the top of the picture in the shadow of the positively biased ohmic electrode. Numerous individual craters can be seen. These gradually merge, towards the bottom, into a more continuous path, but maintain approximately the same depth. This picture is an enlargement of the top track on the disk shown in figure 6 . It should be noted that after extensive pulsing, such tracks generally widen and deepen. They lose the appearance of discrete explosion craters. The walls become more or less continuous and look highly eroded. Figures 3 and 5 are examples of such eroded tracks. Widths and depths of $\sim 50 \mu \mathrm{m}$ have occasionally been observed.

The second basic type of track appears to be continuous rather than a series of discrete explosions. These continuous tracks are clearly shown in figure 10. On a microscopic basis they look like finely ruled lines. Occasionally ones are seen that twist and turn around surface imperfections, but it is difficult to distinguish any discontinuities. However, they do extend slowly across the sample with each succeeding current pulse. They are generally 5 to $10 \mu \mathrm{m}$ wide and of similar 


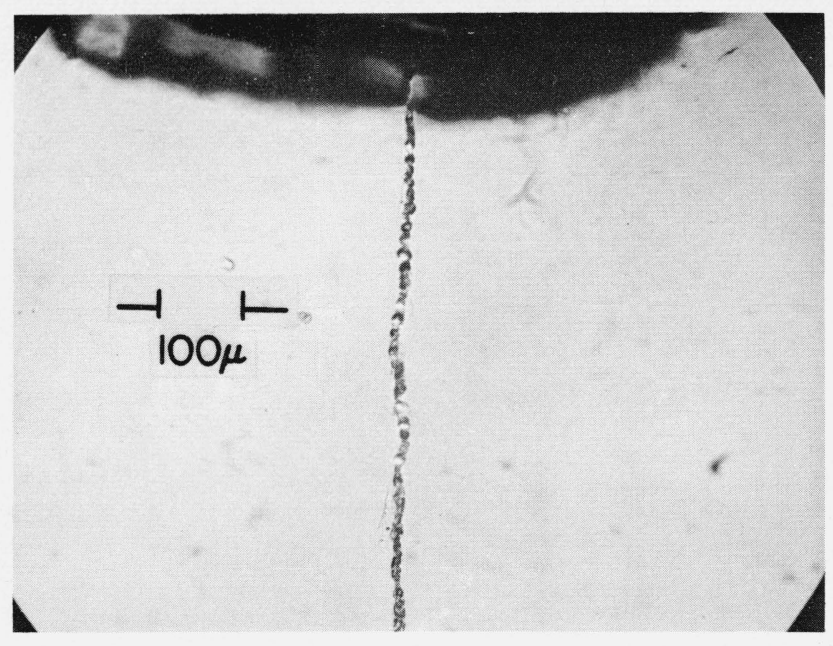

FIGURE 9. An enlargement of a portion of a track shown in figure 6 revealing the explosion craters.

Individual craters are $\sim 10 \mu \mathrm{m}$ in diameter.

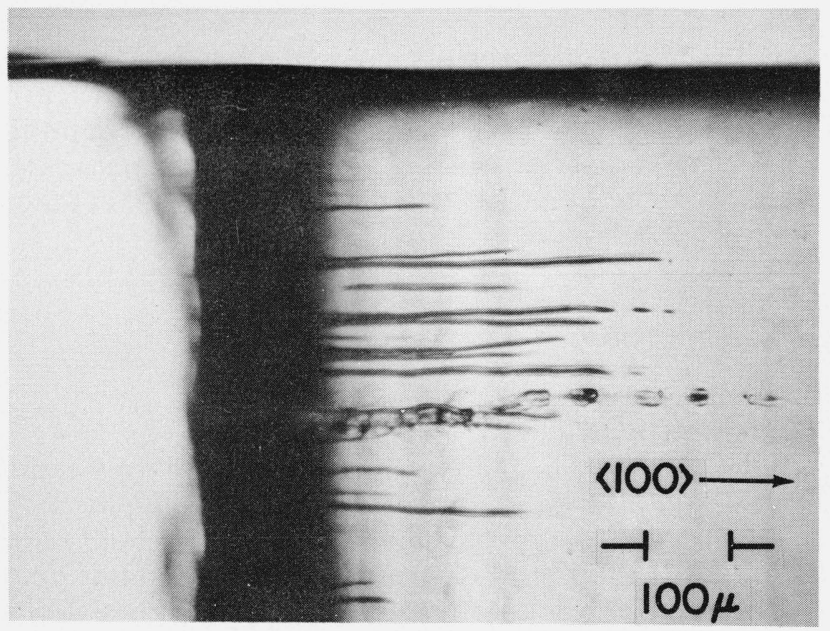

Figure 10. "Continuous" tracks on a $10 \Omega \mathrm{cm}$ p-type $\{110\}\langle 100\rangle$ oriented linear junction sample.

Tracks started in the high field region, near the raised ohmic electrode (left side of figure). An "explosion" type track is in the middle of the group. The tracking threshold was 14 $\mathrm{kV} / \mathrm{cm}, 7.5 \times 10^{3} \mathrm{~A} / \mathrm{cm}^{2}, 0.4 \mu \mathrm{s}$ pulse length, at $300 \mathrm{~K}$

depth. Continued pulsing extends the length, increases the number of individual tracks, but does not appreciably increase their depth. Both types of tracks can occur together, and one of the explosion type appears near the center of figure 10. Note how the two types of tracks snake around one another. They do not merge but go their own individual ways.

There are many unusual configurations that tracks may take. Outstanding among these are parallel tracks that turn in toward themselves and propagate perpendicular to both the field and the current! Figure 11 is an example of these. It is a greatly enlarged portion of a $\{100\}\langle 100\rangle$ sample, showing only the raised ohmic electrode region (region $\mathrm{A}$ of fig. la). These tracks originate at the alloyed aluminum ohmic contact, and progress towards the edge

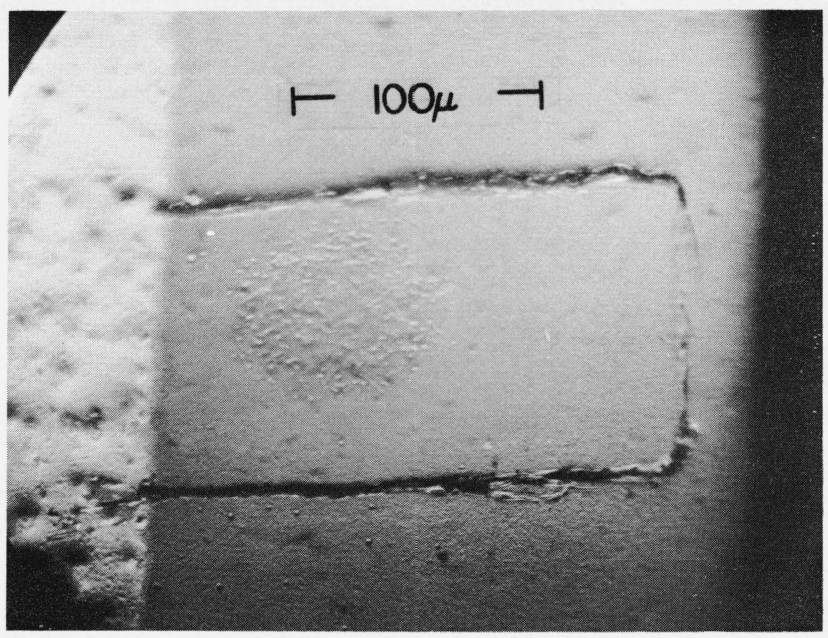

FIGURE 11. Enlargement of the ohmic elecurode area [region $A$ of fig. 1(a)] of a $10 \Omega \mathrm{cm}$ p-type $\{100\}\langle 100\rangle$ junction sample showing tracks that turn in on themselves.

The white material on the left side of the picture is the alloyed aluminum electrode. (It was evaporated about $5^{\circ}$ off perpendicular.)

of region $\mathrm{B}$ ignoring the large surface imperfection between them. At the edge, instead of moving downward toward the higher field region $B$ (which would be in a $\langle 100\rangle$ direction), they turn at right angles to the field (also in a $\langle 100\rangle$ direction) and join together.

Tracks occasionally start in the thick, electroded, region A (fig. la) when alloyed aluminum is used as the ohmic contact. They are only rarely observed there with diffused boron ohmic contacts. The alloyed aluminum apparently creates defects that favor the initiation of tracks. This is so very prominent that whenever alloyed aluminum ohmic contacts are applied to a flat (planar) $\langle 100\rangle$ sample, without raised or bulbous ends, tracks will always originate at the edge of the aluminum, and with very low energy thresholds. There have been cases in which the aluminum and the underlying $\mathrm{Si}-\mathrm{Al}$ alloy material were removed by etching, to facilitate some additional processing step. However, later when the structure was subjected to hot carrier pulses, tracking originated from that same region, indicating that the defects had propagated far into the material. Also, electron microprobe measurements showed that electromigration of aluminum from planar contacts occurs in the tracks. In one case traces of aluminum extended $200 \mu \mathrm{m}$ from the edge of the contact.

\subsection{Tracking Threshold Energies and Conditions}

Table 1 compares the averaged tracking thresholds of a number of linear samples having the dimensions of figure la. The orientations and contact characteristics are indicated. In order to present a meaningful comparison, the values given are all for $0.4 \mu \mathrm{s}$ pulse length at $300 \mathrm{~K}$ and with samples immersed in a fluorocarbon heat exchange liquid. However, other samples have been observed to track with pulse lengths as short as $0.1 \mu \mathrm{s}$. Long pulse lengths $(>1 \mu \mathrm{s})$ can also produce tracking, but heating generally be- 
TABLE 1. Minimum applied impulse to produce tracking on linear samples at $300 \mathrm{~K}$, immersed in a fluorocarbon electrically insulating, heat exchange liquid

All pulse lengths were $0.4 \mu \mathrm{s},(\mathrm{Jn})$ indicates a junction sample, (ohmic) indicates noninjecting contacts, and (planar) indicates flat sample.

Sample dimensions are those of figure la (except Planar).

\begin{tabular}{|c|c|c|c|c|c|c|}
\hline No. of samples in average & $\begin{array}{l}\text { Resistivity } \\
\Omega \mathrm{cm}\end{array}$ & $\begin{array}{l}\text { Bulk carrier } \\
\text { type and contact }\end{array}$ & $\begin{array}{l}\text { Surface plane and } \\
\text { field orientation }\end{array}$ & $\begin{array}{c}\text { Peak } \\
\text { current } \\
\text { density } \\
\mathrm{A} / \mathrm{cm}^{2}\end{array}$ & $\begin{array}{l}\text { Field } \\
\text { strength } \\
\mathrm{kV} / \mathrm{cm}\end{array}$ & $\begin{array}{l}\text { Impulse energy } \\
\text { density } \mathrm{b}^{\mathrm{J}} / \mathrm{cm}^{3}\end{array}$ \\
\hline $5 \ldots \ldots \ldots$ & 100 & $\mathrm{P}(\mathrm{Jn})$ & $\{100\}\langle 100\rangle$ & $2 \times 10^{3}$ & a 60 & 24, Nontrack ${ }^{\mathrm{c}}$ \\
\hline $10 \ldots \ldots \ldots \ldots \ldots \ldots \ldots \ldots \ldots \ldots \ldots \ldots \ldots \ldots \ldots \ldots$ & 10 & $\mathrm{P}(\mathrm{Jn})$ & $\{100\}$ and $\{110\},\langle 100\rangle$ & $6 \times 10^{3}$ & 15 & 18 \\
\hline 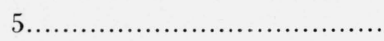 & 10 & $\mathrm{P}(\mathrm{Jn})$ & $\{100\}\langle 110\rangle$ & $22 \times 10^{3}$ & 19 & 85 \\
\hline $5 \ldots \ldots \ldots \ldots \ldots \ldots$ & 10 & $\mathrm{P}(\mathrm{Jn})$ (planar) & $\{100\}\langle 100\rangle$ & $3.5 \times 10^{3}$ & 6.5 & 3.5 \\
\hline 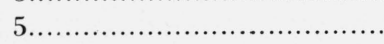 & 10 & $\mathrm{P}(\mathrm{Jn})$ & $\{110\}\langle 111\rangle$ & $25 \times 10^{3}$ & a 32 & 160, Nontrack ${ }^{\mathrm{c}}$ \\
\hline $4 \ldots \ldots \ldots \ldots \ldots \ldots \ldots \ldots \ldots \ldots \ldots \ldots \ldots \ldots \ldots \ldots$ & 1 & $\mathrm{P}(\mathrm{Jn})$ & $\{100\}\langle 100\rangle$ & $50 \times 10^{3}$ & 16 & 160, Nontrack $^{\mathrm{c}}$ \\
\hline $2(3$ more nontrack $) \ldots$. & 1 & $\mathrm{P}$ (ohmic) & $\{100\}\langle 100\rangle$ & $13 \times 10^{3}$ & a 32 & 165 \\
\hline $3(3$ more nontrack)............ & 1 & $\mathrm{~N}($ ohmic $)$ & $\{100\}\langle 100\rangle$ & $7 \times 10^{3}$ & a 32 & 85 \\
\hline
\end{tabular}

${ }^{a}$ Maximum field available for this particular test.

${ }^{b}$ Calculated on the basis of square current wave shape for ohmic samples and triangular shape (figure $2 \mathrm{E}$ ) for junction (minority carrier injection) samples.

c These samples did not track at the indicated high voltage and current density. They exploded at a slightly higher impulse energy density. They are included in this table to compare the largest number of sample orientations and resistivities.

comes severe before the minimum-voltage trackingthreshold is reached. Samples have also been tracked at $77 \mathrm{~K}$ (energy thresholds slightly lower, see fig. 2), and in air at $300 \mathrm{~K}$ (thresholds approximately the same as in liquid but heating was evident).

Samples having different resistivities, orientations, carrier types, and nature of their contacts (ohmic or injecting), display different tracking thresholds. Certain orientations and resistivities will not track, but are thermally destroyed when the power is raised to sufficiently high levels. Maximum nondestructive data on some of these (indicated as nontrack) are included in table 1 for comparison purposes, to show the enormous power such samples can absorb without tracking.

From the data of table 1 it is apparent that the minimum bulk tracking threshold (not originating at planar contacts) is for samples with applied fields $\sim 15 \mathrm{kV} / \mathrm{cm}$ oriented in a $\langle 100\rangle$ direction, and having current densities $\sim 6000 \mathrm{~A} / \mathrm{cm}^{2}$. From the 100 and $1 \Omega \mathrm{cm}$ linear junction-sample data, it is apparent that tracking does not occur unless both the current density and the field (or their $\langle 100\rangle$ components) approximately meet these conditions at the same time. Thus, appropriately longer $1 \Omega \mathrm{cm}$ junction samples (where the same field will produce less current density, due to minority carrier decay) or shorter $100 \Omega \mathrm{cm}$ ones can be made to track. This length effect has been tentatively verified on several samples. However, in order to avoid ambiguity, all data presented in this paper are for samples having the dimensions of fig. 1 . $P$-type disk samples of 1,10 and $100 \Omega \mathrm{cm}$ resistivity (with the ohmic contact in the center and junction on the perimeter) will all track, because the correct combination of $\mathrm{E}$ and $\mathrm{J}$ will always occur at some point as the field is increased. Likewise, $\langle 100\rangle$ oriented planar junction samples the same dimensions as figure la except that region $\mathrm{B}$ has the same thickness as $\mathrm{A}$ and $\mathrm{C}$ ) will always track. The current density and field strength in the immediate vicinity of the electrode edge will meet the necessary conditions as the field is increased. In fact, the primary consideration for tracking in planar structures is the current density per unit length of forward edge of the contact. The appropriate local field is created by the current. A critical value of approximately $30 \mathrm{~A} / \mathrm{cm}$ will produce tracking from alloyed-aluminum planar ohmic contacts on $\langle 100\rangle$ oriented, $10 \Omega \mathrm{cm} p$-type junction samples, with the dimensions of figure la.

Tracking has not been observed on $10 \Omega \mathrm{cm} n$-type linear junction diodes. The large current densities $\left(\sim 5000 \mathrm{~A} / \mathrm{cm}^{2}\right)$ that are necessary for tracking are difficult to obtain, except with disks. Graphs of linear diode $\mathrm{I}-\mathrm{V}$ characteristics resemble the usual $n$-type hot carrier saturation, even though significant injection is present near the junction, as indicated from probe measurements. Presumably the lifetime of the injected holes is too short to permit them to traverse the entire length of the sample, thus limiting the current. Details of this will be investigated at a later date. As the pulse power is increased the samples thermally explode. However, examination of the resulting fragments of $\langle 100\rangle$ oriented linear samples frequently reveal small tracks in the $\langle 100\rangle$ direction (parallel to the field). These occur in the process of the explosion. $N$-type disks (fig. lb) track diffusely, but with a general $\langle 100\rangle$ orientation. In these cases the $n$-type tracking threshold energy was similar to that for comparable $p$-type disk specimens.

A number of $100 \Omega \mathrm{cm} p$-type linear samples, with the dimensions of figure la were studied but none has been observed to track. As with the $10 \Omega \mathrm{cm} n$-type samples, the maximum attainable current density is too low. Eventually, of course, as the power is increased the sample will thermally explode.

Table 1 presented data in terms of the average applied fields. This gives meaningful comparisons for samples with identical (fig. la) configurations but can 
be misleading for variations such as planar electrodes, shorter samples, etc. Therefore, pulse probe measurements were made. For junction samples with figure la dimensions, and with a typical $\langle 100\rangle$ oriented tracking field of $15 \mathrm{kV} / \mathrm{cm}$ applied, a peak field of 1.5 times the average applied field was observed. This peak was approximately $0.5 \mathrm{~mm}$ (in region $\mathrm{B}$, fig. la) from the raised ohmic contact region $\mathrm{A}$.

The situation was quite different for planar contacted samples. The field peaked to $\sim 3$ times the average applied field at the edge of the ohmic contact. Thus the local threshold field necessary to produce tracking on $\langle 100\rangle$ oriented samples was similar for both electrode configurations.

Once a track has started, its surface becomes more conductive, and the local field in its vicinity is reduced about 20 percent, as measured with respect to the ohmic contact. The field reduction extends about $50 \mu \mathrm{m}$ in all directions from the track. This was revealed by actually probing in and along growing tracks. Also, once started, a typical track that has reached an equilibrium length will increase that length by $\sim 35 \mu \mathrm{m}$ per $\mathrm{kV}$ increase in local field.

The thermal impulse heating of samples having the dimension of figure la was determined under hot carrier conditions with the specimens immersed in the fluorocarbon heat exchange liquid. The negative temperature characteristics of ohmic contacted samples were used by observing the current decrease from leading to trailing edge of the pulse. This was measured over a wide power range and at many different ambient temperatures. The graph of sample temperature versus applied power yielded a slope of 1 , as anticipated if it is assumed that all heat loss from the sample to the liquid is by way of simple conduction. From this it was established that the pulse temperature rise of the planar samples (table 1) was only $4 \mathrm{~K}$, and' that of the $10 \Omega \mathrm{cm}\{100\}\langle 100\rangle p$-type junction samples $\sim 20 \mathrm{~K}$. The temperature rise of $1 \Omega \mathrm{cm} p$-type ohmic samples was so large $(\sim 200 \mathrm{~K})$ that some thermally generated minority carriers were present and may have contributed to tracking, though not enough to straighten out the tracks (fig. 8). Tracking on ohmic samples occurs at energies only slightly below thermal breakdown.

\subsection{Anisotropic Explosions}

As shown above, the power required to produce tracking is anisotropic with crystallographic orientation. Since tracking represents a partial destruction of the sample, it is reasonable to assume that catastrophic destruction (exploding samples) is a related phenomenon and is also anisotropic. Figure 9 demonstrated that one mode of tracking is propagated by a series of tiny explosions. Such tracking was studied near its threshold power level. One can readily visualize a situation in which a single higher powered pulse is applied, and a large portion of the sample disintegrates as one macroscopic track. A few selected $\langle 100\rangle$, and $\langle 111\rangle$ junction and ohmic samples have been intentionally destroyed in one pulse. Microscopic observation of the many small pieces of the $\langle 100\rangle$ junction samples indicated that part of the surface must have erupted in the manner of an exploding wire (macroscopic track). Ohmic contacted samples and $\langle 111\rangle$ junction samples typically explode into several large pieces with no evidence of disintegration.

Partial explosions are apt to occur while slowly increasing the power level above the tracking threshold. A usual result of this is the blasting off of a chip from the edge of the sample. Typically this occurs in region B about $0.5 \mathrm{~mm}$ from the raised ohmic contact. This is the region of maximum local field as indicated by probing. A photograph of a partial explosion is given in figure 12 . This $\{100\}\langle 100\rangle$ oriented junction sample developed a deep track and then blasted off the chip. The edges of the hole clearly show $\{110\}$ cleavage planes, attesting to the internal force of the tracking phenomenon. This track and hole are the only damage on an otherwise perfect sample, showing how localized such effects can be.

The first impression one might have is that samples simply disintegrate from thermal impulse. This undoubtedly has a contributory effect at high power. levels. Therefore, it was necessary to separate the thermal impulse contribution from the macroscopic tracking effect. This was done by exploding $\langle 111\rangle$ oriented samples. Such samples do not track, except occasionally in the actual process of exploding. Thus, the impulse required to explode these can be used as an upper or thermal breakdown limit. Table 2 compares the energy required to explode both tracking and nontracking samples. It includes the approximate temperature of the samples at the explosion energy. The relatively large energy difference required to explode $\langle 111\rangle$ junction versus ohmic samples is not understood. However, the $\langle 111\rangle$ junction sample results appear to be more relevant in defining a thermal breakdown limit for anisotropic tracking and explosions on other junction samples.
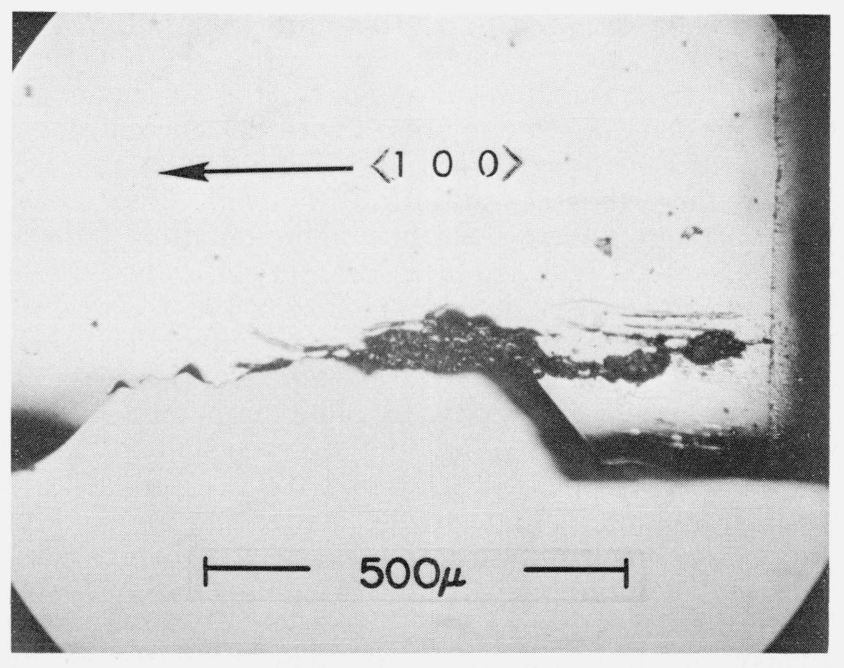

FigURE 12. A section of a $10 \Omega \mathrm{cm}$ p-type $\{100\}\langle 100\rangle$ junction linear sample with a piece exploded out of its side. 
TABLE 2. Applied power impulse required to explode silicon linear samples that are immersed in a fluorocarbon heat exchange liquid, at $300 \mathrm{~K}, 0.4$ or $0.25 \mu \mathrm{s}$ pulse length, 2 pulses per second

Sample dimensions are those of figure la.

\begin{tabular}{|c|c|c|c|c|c|}
\hline $\begin{array}{l}\text { No. of } \\
\text { samples in } \\
\text { average }\end{array}$ & $\begin{array}{l}\text { Resistivity } \\
\Omega \mathrm{cm}\end{array}$ & $\begin{array}{l}\text { Surface plane } \\
\text { and field } \\
\text { orientation }\end{array}$ & $\begin{array}{c}\text { Bulk carrier } \\
\text { type and } \\
\text { contact }\end{array}$ & $\begin{array}{c}\text { Approximate } \\
\text { temp. rise }^{\text {a }} \\
\text { preceeding } \\
\text { explosion } T K\end{array}$ & $\begin{array}{l}\text { Impulse energy } \\
\text { Density }{ }^{\mathrm{b}} \mathrm{J} / \mathrm{cm}^{3}\end{array}$ \\
\hline 4 & 10 & $\{100\}\langle 100\rangle$ & $\mathrm{P}(\mathrm{Jn})$ & 50 & 40 \\
\hline 4 & 10 & $\{100\}\langle 110\rangle$ & $\mathrm{P}(\mathrm{Jn})$ & 140 & 120 \\
\hline 6 & 10 & $\{110\}\langle 111\rangle$ & $\mathrm{P}(\mathrm{Jn})$ & 290 & 240 \\
\hline 4 & 1 & $\{110\}\langle 111\rangle$ & N(ohmic) & 210 & 175 \\
\hline
\end{tabular}

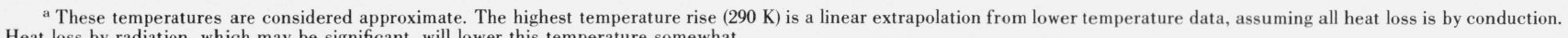
Heat loss by radiation, which may be significant, will lower this temperature somewhat.

See footnote b, Table 1 .

The anisotropic explosion results are clearly in agreement with those of table 1 for tracking. It is evident from table 2 that $\langle 110\rangle$ and $\langle 111\rangle$ linear samples require significantly more energy to explode than do the $\langle 100\rangle$ linear samples, and the differences are far too great to be attributed to heating effects.

\section{Discussion}

From the experimental evidence of table 1 it is clear that both high fields and high current densities are required to initiate tracking. It is assumed that each individual track starts on a defect, as a result of its crystallographic weakness or its perturbation of the local electric field. Once started, the local field is modified somewhat by the first crater (as indicated by probe measurements). Subsequent craters can then form without requiring a defect in the material.

However, there is no apparent reason why the track should propagate in the $\langle 100\rangle$ direction when the field is applied in a different direction. The $\langle 100\rangle$ direction is not known to have excessive defects propagating along it. Nor is it the weakest mechanical direction. Cleavage in silicon occurs along $\{110\}$ planes and Poissons ratio has a sharp minimum there [7]. Oswald [8] has studied the fracture patterns for silicon when samples were subjected to massive electrical energy impulses. He exposed his specimens to $40 \mathrm{~ns}, 2.2 \mathrm{MeV}$ electron beam pulses and found that fracture occurs along $\langle 111\rangle$ directions. The energy was considered to be thermal in nature, and the samples were shown to crack by brittle fracture. There is no evidence that the tracks are related to the present crystal growth direction $(\langle 111\rangle$ or $[2 \overline{11}])$, as Morozov [2] suggested for germanium. With mechanical and thermal properties ruled out as the source of anisotropic tracking, we will examine the various electronic properties of the material in an effort to understand the phenomena.

Anisotropy in the high voltage breakdown of insulator crystals has been known for years [9, 10, 11]. Depending on the particular crystal, the field strength, and the orientation, preferential paths have been observed along $\langle 100\rangle,\langle 110\rangle$, and $\langle 111\rangle$ crystallographic directions. Generally each of the three paths can be obtained on a single material at different temperatures
[12] or with overvoltage. Breakdown voltages for these effects on typical insulators, such as alkali halides, are $\sim 10^{6} \mathrm{~V} / \mathrm{cm}$. In general, oriented breakdowns of insulators are only observed when a crystal is placed between a point and a plane contact and immersed in a liquid insulating medium. Such an electrode arrangement does not remotely resemble the situation in the present work. Also, the high current density necessary for silicon tracking is clearly not a requirement for insulator tracking. In addition, no change in tracking orientation has presently been observed at different temperatures or with overvoltage.

During the present experiments the samples were immersed in liquid nitrogen (breakdown $\sim 10^{6} \mathrm{~V} / \mathrm{cm}$ ) or a fluorocarbon insulating liquid (breakdown $\sim 2 \times 10^{5}$ $\mathrm{V} / \mathrm{cm}$ ). Since the dielectric strength of silicon is very high, $\sim 5 \times 10^{5} \mathrm{~V} / \mathrm{cm}$ (from reverse bias junction data), ordinary high voltage arcing within the silicon sample or in the surrounding media is not possible with the present applied or local fields. In addition, several samples were further insulated with $8000 \mathrm{~A}$ of grown silicon oxide over the entire surface. This film raised the threshold somewhat but the samples still tracked. Therefore, considering all of these facts, it appears that there is no connection between semiconductor tracking and the well-known tracking in insulators.

Warm and hot majority carrier conduction anisotropy in $n$-type silicon and germanium has been known for many years [13-18]. The presently accepted theory of conduction anisotropy in $n$-type silicon attributes the anisotropy primarily to the scattering of electrons from hot valleys (major axis of the energy ellipsoid perpendicular to the field) into cold valleys (major axis parallel to the field). This takes place with the spontaneous emission or absorption of high energy intervalley phonons. The effect is typically observed in silicon as a relative decrease in conductivity when the field is oriented in a $\langle 100\rangle$ direction compared to the conductivity along $\langle 111\rangle$ or $\langle 110\rangle$ directions. This conductivity difference is maximum for applied fields in the order of several hundred volts per centimeter and decreases as the field is further increased. The difference also decreases as the carrier concentration increases, and all but vanishes when carrier densities and fields approach those required for the present tracking. 
We have established that both high fields and high current (carrier) densities are necessary to produce the observed tracking phenomenon. Since $\langle 111\rangle$ junction samples do not track (all valleys are equivalent), it appears that an intervalley exchange of minority carrier electrons is necessary for oriented tracking. However, majority carrier holes are also necessary for low tracking thresholds as seen from the $1 \Omega \mathrm{cm} n$-type ohmic data of table 1. A further complication exists because the electrons are hot and the holes are merely warm. In addition, the field strength is not uniform along the sample, as determined by probe measurements. The carrier density is very high so that intercarrier scattering as well as lattice and intervalley scattering should be considered. Thus, the theoretical situation for hot carrier induced anisotropic tracking is much more difficult than majority carrier conduction anisotropy.

If the accepted conduction anisotropy theory for $n$-type silicon were applicable to the present work it would imply that the current, and thus the total energy, was lowest in the $\langle 100\rangle$ direction of tracking. This does not present any obvious mechanism for tracking. One might then assume that there was a very high current (perhaps filamentary) flowing along the $\langle 100\rangle$ track direction. Examination of figures 3, 7, and especially the converging tracks of figure 11 show that tracks can propagate in $\langle 100\rangle$ directions in which high current cannot uniquely flow. The actual driving force for tracking must derive its energy from the high field and high current density. But, the force must also be independent of their direction in order to explain figures 3,7 , and 11 . It should also manifest itself at the surface.

One theory that might conceivably be applicable to anisotropic tracking was given by Rashba [19]. He states that since the ellipsoids of a many valley semiconductor are inclined at different angles to the surface, an electric field parallel to the surface plane will scatter more electrons toward that surface than are scattered back. At low temperatures this can significantly increase the carrier concentration within $\sim 10 \mu \mathrm{m}$ of the surface and in an anisotropic manner. The theory is not completely developed. It is only solved in the case of a weak, constant electric field, for a four valley semiconductor, and would be exceedingly difficult to adapt to a two carrier, hot carrier situation. Its primary merit is that it proposes an increased concentration of carriers in the surface region of the sample, where tracking is observed.

Kornreich, Callen, and Gundjian [20] have recently reported the theory of "current striction." Not enough information has yet been published to evaluate it under the present two carrier, hot carrier situation. However, it is a new mechanism of electrostriction in a many valley semiconductor which should yield oriented characteristics. The effect on silicon is about 100 times larger than could be expected from conventional electrostriction mechanisms and is larger still for germanium. "The current striction effect lowers the energy of those valleys with major axes (which are along $\langle 100\rangle$ ) parallel to the field, and thereby concen- trates electrons into such valleys. This effect appears to be at least qualitatively similar to the tracking effect" [21]. In addition it would result in $\langle 111\rangle$ oriented forces in germanium, as observed by Morozov. The current striction authors also indicated that they were extending their work to $p$-type material and such work, combined with their original paper, could yield a quantitative insight into why $p$-type bulk, with minority carriers present, produces by far the lowest thresholds for anisotropic tracking.

The obvious merit of the "striction" mechanism is that in silicon it produces oriented forces along $\langle 100\rangle$ directions that need not be parallel to the applied field. The forces should be minimum with the field in a $\langle 111\rangle$ direction, and would not produce tracking. Since the surface is normally considered the weakest portion of a sample, any large internal force will manifest itself there, and cause breakdown at the weakest point, which is a defect. Propagation would follow the direction of the force, the $\langle 100\rangle$. Anisotropic explosions (macroscopic tracks) would be an expected consequence of such a "striction" mechanism.

Note added in the proof: Since this paper was submitted, a new mechanism, "Electronic Volume Effect in Semiconductors", has been described [W. B. Gauster, Bull. Am. Phys. Soc. 14, 312 (1969)]. This mechanism is a two carrier effect, related to photo striction, and may be applicable to the present tracking phenomena.

\section{Conclusions}

The present paper has described the topological features of $\langle 100\rangle$ oriented anisotropic tracking on silicon. The available evidence from tables 1 and 2 indicates that low threshold energy anisotropic tracking, and sample explosions are a two-carrier, hotcarrier phenomena. There is no obvious mechanical or electronic reason why tracks should propagate preferentially along $\langle 100\rangle$ directions. The present hot carrier anisotropy theory fails to suggest an answer to this tracking. It appears that the explanation of both anisotropic tracking and explosions awaits further development of a two carrier "current striction" mechanism.

Some breakdowns in Gunn and avalanche devices $[22,23]$ strongly resemble tracks; similar situations have been observed in our laboratory. The structure of high-current-density planar devices makes them obvious targets for failures due to tracking, and narrow electrode spacings or other probable breakdown paths should be designed along $\langle 111\rangle$ or $\langle 110\rangle$ directions to avoid failure. Some additional protection can be obtained by covering surfaces with silicon dioxide. Also alloyed contacts should be avoided when possible. Another device failure mechanism, second breakdown, [24] should be reinvestigated for a possible orientation dependance. Examination of failures in certain high current, high voltage, $p-n-p-n$ devices [25] and similar $n^{+}-p$ diodes [26] has yielded oriented breakdown patterns in the vicinity of the junction, 
or on its surface, that look similar to those of germanium [2]. Under any conditions the possibility of an orientation dependance of device breakdown should be considered, even though normal performance of the device may have been shown to be independent of crystallographic orientation.

The author is indebted to M. Bullis, E. Farabaugh, and J. Scales for valuable discussions. K. Leedy made the hot-carrier sample-temperature measurements. Phosphorous and boron diffusions were done in the facilities of Harry Diamond Laboratories of the Army Materiel Command.

\section{References}

[1] Gunn, J. B., J. Electronics 2, 87 (1956).

[2] Morozov, A. I., Fizika Tverdogo Tela 2, 620 (1960).

[3] Harman, G. G., Bull. Am. Phys. Soc. 13, 406 (1968).

[4] Harman, G. G., Appl. Phys. Lett. 9, 207 (1966).

[5] The general crystallographic orientation shorthand to be used is as follows: $\{111\}$ indicates the surface plane of the slice or sample, $\langle 110\rangle$ indicates the direction of the longest dimension of a rectangular sample and thus is the orientation of the applied field. A combination of these, $\{100\}\langle 100\rangle$, completely describes the crystallography of rectangular samples. Only the surface plane is indicated for disk samples except where a tracking direction is discussed.

[6] Mayer, J. W., Baron, R., and Marsh, O. J., Phys. Rev. 137, A286 (1965).
[7] Wortman, J. J., and Evans, R. A., J. Appl. Phys. 36, 153 (1965).

[8] Oswald, R. B., Bull. Am. Phys. Soc. 11, 363 (1966); IEE Trans. Nuclear Sci. NS-13, 63 (1966).

[9] Inge, L., and Walther, A., Z. Phys. 64, 830 (1930).

[10] von Hippel, A., Z. Phys. 67, 707 (1931); 68, 309 (1931); 75, 145 (1932).

[11] Offenbacker, E. L., and Callen, H. B., Phys. Rev. 90, 401 (1953).

[12] Davisson, J. W., Phys. Rev. 70, 685 (1946).

[13] Shibuya, M., Phys. Rev. 99, 1189 (1955).

[14] Sasaki, W., Shibuya, M., Mizuguchi, K., and Hatoyama, G. M., J. Phys. Chem. Solids 8, 250 (1959).

[15] Jørgensen; M. H., Meyer, N. I., and Schmidt-Tiedemann, K. J., Proc. 7th Intl. Conf. on Semiconductors, Paris 1964, page 457.

[16] Kastner, P., Roth, E.-P., and Seeger, K., Zeit. fur Physik 187, 359 (1965).

[17] Gibbs, W. E. K., J. Phys. Chem. Solids 25, 247 (1964).

[18] For a recent review see, B. R. Nag, Solid State Elect. 10, 385 (1967).

[19] Rashba, E. I., J. Exptl. Theoret. Phys. (USSR) 48, 1427 (1965)

[20] Kornreich, P., Callen, H. B., and Gundjian, A., Phys. Rev. 161, 815 (1967)

[21] Callen, H. B., private communication.

[22] Jeppsson, B., and Marklund, I., Elect. Lett. 3, 213 (1967).

[23] Schenck, J. F., and Midford, T. A., IEEE Trans. Elect. Devices ED-14, 619 (1967)

[24] Schafft, H. A., Proc. IEEE 55, 1272 (1967).

[25] Localized Defects and Hot Spots in High Voltage pnpn Inverter Switches. General Electric Co. contract NO bsr-87648, Mod. Nr. 9, AD 489, 530; 810, 820; 804, 491 (released by the Clearinghouse for Federal Scientific and Technical Information, Springfield, Va. 22151).

[26] Thomson, I., and Wilkinson, E. L. G., Proc. Symposium on Test Methods and Measurements of Semiconductor Devices Page 101, Budapest, April 25, 1967.

(Paper 73A3-556) 